\title{
Generalized magneto-thermoviscoelasticity in a perfectly conducting thermodiffusive medium with a spherical cavity
}

\author{
Ashraf M Zenkour ${ }^{1,2, *}$, Ebraheem O Alzahrani ${ }^{1}$ and Ahmed E Abouelregal ${ }^{3,4}$ \\ ${ }^{1}$ Department of Mathematics, King Abdulaziz University, P.O. Box 80203, Jeddah 21589, Saudi Arabia. \\ ${ }^{2}$ Department of Mathematics, Kafrelsheikh University, Kafr El-Sheikh 33516, Egypt. \\ ${ }^{3}$ Department of Mathematics, Mansoura University, Mansoura 35516, Egypt. \\ ${ }^{4}$ Department of Mathematics, College of Science and Arts, Aljouf University, El-Qurayat, Saudi Arabia. \\ *Corresponding author.e-mail: zenkour@hotmail.com
}

In this work, the effects of viscosity and diffusion on thermoelastic interactions in an infinite medium with a spherical cavity are studied. The formulation is applied to the generalized thermoelasticity based on the theory of generalized thermoelastic diffusion with one relaxation time. The surface of the spherical cavity is taken to be traction free and subjected to both heating and external constant magnetic field. The solution is obtained in the Laplace transform domain by using a direct approach. The solution of the problem in the physical domain obtained numerically using a method based on Fourier expansion techniques. The temperature, displacement, stress, concentration as well as the chemical potential are obtained and represented graphically. Comparisons are made within the theory in the presence and absence of viscosity and diffusion.

\section{Introduction}

The classical theory of thermoelasticity has been generalized and modified into various thermoelastic models that run under the label of 'hyperbolic thermoelasticity'. The notation hyperbolic reflects the fact that thermal waves are modelled, avoiding the physical paradox of the infinite propagation speed of the classical model. At present, there are several theories of hyperbolic thermoelasticity. The first theory is developed by Lord and Shulman (1967) who obtained a wave-type heat equation by postulating a new law of heat conduction to replace the classical Fourier law. This new law comprises the heat flux vector as well as its time derivative. It also comprises a new constant that acts as a relaxation time. The second theory is developed by Green and Lindsay (1972) to comprise two constants that act as relaxation times. This theory modifies not only the heat conduction equation but also all the equations of coupled theory. These two theories (Lord and Shulman 1967; Green and Lindsay 1972) ensure finite speeds of propagation for heat wave.

Diffusion can be defined as the migration of particles from regions of high concentration to regions of lower concentration. The recent interest in the study of this phenomenon is due to its many industrial applications. Nowacki (1974a, b, c) developed the theory of thermoelastic diffusion in which the coupled thermoelastic model is used. This implies infinite speed of propagation of thermoelastic waves. Sherief et al. (2004) developed the theory of generalized thermoelastic diffusion that predicts finite speeds of propagation for thermoelastic and diffusive waves. Sherief and Saleh (2005) worked on a problem of a thermoelastic half-space with a permeating substance, in contact with the

Keywords. Thermoviscoelasticity; spherical cavity; thermoelastic diffusion; Laplace transform. 
bounding plane in the context of the theory of generalized thermoelastic diffusion.

The observed attenuation of seismic waves on earth, an important source of information regarding the composition and state of the deep interior cannot be explained by assuming the earth to be an elastic solid. Keeping this fact in mind, several problems on wave propagation in a linear viscoelastic solid have been discussed by many researchers. Also with the rapid development of polymer science and plastic industry, as well as the wide use of materials under high temperature in modern technology, the theoretical study and application in viscoelastic materials has become an important task for solid mechanics. The theory of thermoviscoelasticity and the solutions of some boundary value problems of thermoviscoelasticity are investigated by Ilioushin and Pobedria (1970). The works of Tanner (1988) and Huilgol and Phan-Thien (1997) have made great strides in the last decade in finding solutions for boundary value problems for linear viscoelastic materials including temperature variations in both quasi-static and dynamic problems.

Xia et al. (2009) studied the dynamic response of an infinite body with a cylindrical cavity whose surface suffers thermal shock using finite element method. Roychoudhuri and Mukhopadhyay (2000) studied the effect of rotation and relaxation times on plane waves in generalized thermoviscoelasticity. Roychoudhuri and Banerjee (1998) investigated the magneto-thermoelastic interactions in an infinite viscoelastic cylinder of temperature ratedependent material subjected to periodic loading. Spherically-symmetric thermoviscoelastic waves in a viscoelastic medium with a spherical cavity was discussed by Banerjee and Roychoudhuri (1995). Ezzat and El-Karamany (2002) established the uniqueness and reciprocity theorems for generalized thermoviscoelasticity with two relaxation times. The thermoviscoelastic interaction in a homogeneous, infinite Kelvin-Voigt type viscoelastic, thermally-conducting medium due to the presence of periodically varying heat sources has been studied by Kanoria and Mallik (2010). Zenkour et al. (2012) obtained a general solution to the field equations of generalized thermodiffusion in an infinite thermoelastic body with a spherical cavity in the context of the theory of generalized thermoelastic diffusion. The bounding surface of the sphere is subjected to periodic loading and the temperature and chemical potential are assumed to be zero on the curved surface.

The objective of the present work is to determine the components of displacement, stresses, temperature and chemical potential distributions in an isotropic homogeneous magnetothermoviscoelastic infinite medium with a spherical cavity. The problem is solved in the context of the theory of generalized thermoviscoelastic diffusion with one relaxation time. The surface of the spherical cavity is taken to be traction free and subjected to both heating and external constant magnetic field. The solution of the problem is obtained in the transformed domain by using a direct approach without the customary use of potential functions. An exact solution of the problem is first obtained in Laplace transform space. The inversions of Laplace transforms have been carried out numerically. Numerical results predict finite speeds of propagation for thermoelastic and diffusive waves. To investigate the viscosity, magnetic field and diffusions effects, a comparison is made with the results obtained in the thermoelastic problem. Finally, by taking an appropriate material, the results are presented in graphical form to illustrate the problem.

\section{Basic equations}

Let us consider a perfect electric conductor medium in the absence of the displacement current (SI). The linearized Maxwell equations governing the electromagnetic field are given by Ezzat (1997):

$$
\begin{gathered}
\operatorname{curl} \mathbf{h}=\mathbf{J}, \quad \operatorname{curl} \mathbf{E}=-\mu_{0} \frac{\partial \mathbf{h}}{\partial t}, \\
\mathbf{E}=-\mu_{0} \frac{\partial \mathbf{u}}{\partial t} \times \mathbf{H}_{0}, \quad \operatorname{div} \mathbf{h}=0, \quad \operatorname{div} \mathbf{E}=0,
\end{gathered}
$$

where $\mu_{0}$ is the magnetic permeability, $\mathbf{J}$ is the current density vector, $\mathbf{E}$ is the induced electric field, $\mathbf{H}=\mathbf{H}_{0}+\mathbf{h}$ in which $\mathbf{H}_{0}$ is the applied magnetic field and $\mathbf{h}$ is the perturbation occurred in the total magnetic field by induction.

The governing equations for a linear isotropic homogeneous thermoviscoelastic solid with generalized thermodiffusion in the absence of body forces take the following forms (Sherief et al. 2004; Sherief and Saleh 2005):

- The equations of motion have the following form:

$$
\begin{aligned}
\mu^{*} u_{i, j j}+ & \left(\lambda^{*}+\mu^{*}\right) u_{j, i j}-\beta_{1}^{*} \theta_{, i}-\beta_{2}^{*} C_{, i}+F_{i} \\
= & \rho \frac{\partial^{2} u_{i}}{\partial t^{2}}
\end{aligned}
$$

where $u_{i}$ are the components of the displacement vector, $\theta=T-T_{0}$, in which $T$ is the absolute temperature of the medium, $T_{0}$ is the reference uniform temperature of the body chosen such that $\left|\theta / T_{0}\right|=1, C$ is the concentration of the diffusive material in the elastic body, $\rho$ is the density and $F_{i}$ is the components of Lorentz force, whose expression is:

$$
F_{i}=\mu_{0}(\mathbf{J} \times \mathbf{H})_{i} .
$$


The parameters $\lambda^{*}, \mu^{*}, \beta_{1}^{*}$ and $\beta_{2}^{*}$ are defined as:

$$
\begin{array}{ll}
\lambda^{*}=\lambda_{e}\left(1+\alpha_{1} \frac{\partial}{\partial t}\right), & \mu^{*}=\mu_{e}\left(1+\alpha_{2} \frac{\partial}{\partial t}\right), \\
\beta_{1}^{*}=\beta_{1 e}\left(1+\beta_{1} \frac{\partial}{\partial t}\right), & \beta_{2}^{*}=\beta_{2 e}\left(1+\beta_{2} \frac{\partial}{\partial t}\right),(4)
\end{array}
$$

where

$$
\begin{aligned}
& \beta_{1 e}=\left(3 \lambda_{e}+2 \mu_{e}\right) \alpha_{t}, \\
& \beta_{2 e}=\left(3 \lambda_{e}+2 \mu_{e}\right) \alpha_{c}, \\
& \beta_{1}=\left(3 \lambda_{e} \alpha_{1}+2 \mu_{e} \alpha_{2}\right) \frac{\alpha_{t}}{\beta_{1 e}}, \\
& \beta_{2}=\left(3 \lambda_{e} \alpha_{1}+2 \mu_{e} \alpha_{2}\right) \frac{\alpha_{c}}{\beta_{2 e}},
\end{aligned}
$$

in which $\lambda_{e}, \mu_{e}$ being Lamés constants, $\alpha_{1}, \alpha_{2}$ are the thermoviscoelastic relaxation times and $\alpha_{t}$ and $\alpha_{c}$ are the coefficients of linear thermal expansion and linear diffusion expansion, respectively.

- Maxwell electromagnetic stress tensor $M_{i j}$ in the cavity results due to the induced fields. It is given by

$$
M_{i j}=\mu_{0}\left(H_{i} h_{j}+H_{j} h_{i}-\delta_{i j} H_{k} h_{k}\right) .
$$

- The energy equation in the context of generalized thermoelastic diffusion with one relaxation time developed by Lord and Shulman (1967), is modified to the form (Sherief et al. 2004):

$$
K \theta_{, i i}=\left(\frac{\partial}{\partial t}+t_{0} \frac{\partial^{2}}{\partial t^{2}}\right)\left(\rho C_{E} \theta+\beta_{1}^{*} T_{0} e+a T_{0} C\right),
$$

where $K$ is the thermal conductivity, $C_{E}$ is the specific heat at constant strain, $a$ is a coefficient describing the measure of thermoelastic diffusion effects, $e=e_{k k}$ is the cubical dilatation and $t_{0}$ is the thermal relaxation time.

- The equation of mass diffusion:

$$
D \beta_{2}^{*} e_{, i i}+D a \theta_{, i i}+\left(\frac{\partial}{\partial t}+t_{1} \frac{\partial^{2}}{\partial t^{2}}\right) C-D b C_{, i i}=0,
$$

where $D$ is the diffusion coefficient or diffusivity, $t_{1}$ is the diffusion relaxation time, $b$ is a coefficient describing the measure of diffusive effects and $e_{i j}$ are the components of the strain tensor.

- The constitutive equations have the form:

$$
\sigma_{i j}=2 \mu^{*} e_{i j}+\delta_{i j}\left(\lambda^{*} e_{k k}-\beta_{1}^{*} \theta-\beta_{2}^{*} C\right),
$$

where $\sigma_{i j}$ are the components of the stress tensor and $\delta_{i j}$ is Kronecker's delta tensor.
- The chemical potential equation has the form:

$$
P=-\beta_{2}^{*} e_{k k}+b C-a \theta,
$$

where $P$ is the chemical potential per unit mass.

It will ensure that the equation satisfied by the concentration of the diffusive material $C$ will also predict finite speed of propagation of matter from one medium to the other.

\section{Formulation of the problem}

Let us consider a homogeneous, isotropic thermoelastic infinite body with a spherical cavity of radius $R$. The spherical polar coordinates $(r, \Theta, \phi)$ are taken for any representative point of the body at time $t$ and the origin of the coordinate system is at the center of the spherical cavity. Let us also assume that the initial state of the medium is quiescent. The surface of the spherical cavity is assumed to be traction free and subjected to heating which is a function of time. The chemical potential is also assumed to be a known function of time on the bounding cavity. All the variables considered will be functions of the radial distance $r$ and the time $t$ only.

Considering radial variations of the medium, the only non-zero displacement component is $u_{r}=$ $u(r, t)$, so that, the component of strain tensor are:

$$
\begin{aligned}
& e_{r r}=\frac{\partial u}{\partial r}, \quad e_{\Theta \Theta}=\frac{u}{r}=e_{\phi \phi}, \\
& e_{r \phi}=e_{r \Theta}=e_{\Theta \phi}=0 .
\end{aligned}
$$

The dilatation $e$ thus, will be

$e=\operatorname{div}(u)=e_{r r}+e_{\phi \phi}+e_{\Theta \Theta}=\frac{\partial u}{\partial r}+\frac{2 u}{r}=\frac{1}{r^{2}} \frac{\partial}{\partial r}\left(r^{2} u\right)$.

The constitutive equations for a spherical symmetric system are given by

$$
\begin{aligned}
\sigma_{r r} & =2 \mu^{*} \frac{\partial u}{\partial r}+\lambda^{*} e-\beta_{1}^{*} \theta-\beta_{2}^{*} C, \\
\sigma_{\Theta \Theta} & =2 \mu^{*} \frac{u}{r}+\lambda^{*} e-\beta_{1}^{*} \theta-\beta_{2}^{*} C,
\end{aligned}
$$

and the chemical potential is

$$
P=-\beta_{2}^{*} e+b C-a \theta .
$$

The equations of heat conduction and mass diffusion are:

$$
\begin{aligned}
& K \nabla^{2} \theta=\left(\frac{\partial}{\partial t}+t_{0} \frac{\partial^{2}}{\partial t^{2}}\right)\left(\rho C_{E} \theta+\beta_{1}^{*} T_{0} e+a T_{0} C\right), \\
& D \beta_{2}^{*} \nabla^{2} e+D a \nabla^{2} \theta+\left(\frac{\partial}{\partial t}+t_{1} \frac{\partial^{2}}{\partial t^{2}}\right) C=D b \nabla^{2} C,
\end{aligned}
$$


where $\nabla^{2}$ is Laplace operator in spherical coordinates

$$
\nabla^{2}=\frac{\partial^{2}}{\partial r^{2}}+\frac{2}{r} \frac{\partial}{\partial r}
$$

Due to the application of the initial magnetic field $\mathbf{H}_{0}$, there results an induced magnetic field $\mathbf{h}=$ $(0,0, h)$ which be small, so that, their products with $u_{i}$ and their derivatives can be neglected for linearization and an induced electric field $\mathbf{E}$. Applying an initial magnetic field vector $\mathbf{H}_{0}=$ $\left(0,0, H_{0}\right)$ to equation (1), one obtains

$$
\begin{aligned}
\mathbf{E} & =\left(0, \mu_{0} H_{0} \frac{\partial u}{\partial t}, 0\right), \quad \mathbf{J}=\left(0,-\frac{\partial h}{\partial r}, 0\right), \\
h & =-H_{0}\left(\frac{\partial u}{\partial r}+\frac{2 u}{r}\right) .
\end{aligned}
$$

The components of Lorentz force can be obtained from equation (18) in the form

$$
F_{r}=\mu_{0}(\mathbf{J} \times \mathbf{H})_{r}=\mu_{0} H_{0}^{2} \frac{\partial}{\partial r}\left(\frac{\partial u}{\partial r}+\frac{2 u}{r}\right) .
$$

The equation of motion, equation (2), then reduces to

$$
\sigma_{r r, r}+\frac{\sigma_{r r}-\sigma_{\Theta \Theta}}{r}+F_{r}=\rho \frac{\partial^{2} u}{\partial t^{2}} .
$$

Thus, from equations (13) and (20), one obtains

$$
\left(\lambda^{*}+2 \mu^{*}+\mu_{0} H_{0}^{2}\right) \frac{\partial e}{\partial r}-\beta_{1}^{*} \frac{\partial \theta}{\partial r}-\beta_{2}^{*} \frac{\partial C}{\partial r}=\rho \frac{\partial^{2} u}{\partial t^{2}} .
$$

Applying the operator $(\partial / \partial r+2 / r)$ to both sides of equation (21), one gets

$$
\left(\lambda^{*}+2 \mu^{*}+\mu_{0} H_{0}^{2}\right) \nabla^{2} e-\beta_{1}^{*} \nabla^{2} \theta-\beta_{2}^{*} \nabla^{2} C=\rho \frac{\partial^{2} e}{\partial t^{2}} .
$$

For convenience, the following non-dimensional quantities are used

$$
\begin{aligned}
r^{\prime} & =c_{1} \eta r, \quad u^{\prime}=c_{1} \eta u, \quad \theta^{\prime}=\frac{\beta_{1 e}}{\lambda_{e}+2 \mu_{e}} \theta, \\
P^{\prime} & =\frac{P}{\beta_{2 e}}, \quad t^{\prime}=c_{1}^{2} \eta t, \quad t_{0}^{\prime}=c_{1}^{2} \eta t_{0}, \quad t_{1}^{\prime}=c_{1}^{2} \eta t_{1}, \\
C^{\prime} & =\frac{\beta_{2 e}}{\lambda_{e}+2 \mu_{e}} C, \quad \sigma_{i j}^{\prime}=\frac{1}{\lambda_{e}+2 \mu_{e}} \sigma_{i j}, \\
q_{r}^{\prime} & =\frac{\beta_{1}}{K c_{1}\left(\lambda_{e}+2 \mu_{e}\right)} q_{r}, \quad M_{i j}^{\prime}=\frac{M_{i j}}{\mu},
\end{aligned}
$$

where $\eta=\frac{\rho C_{E}}{K}, c_{1}=\sqrt{\frac{\lambda_{e}+2 \mu_{e}}{\rho}}$ is the speed of propagation of isothermal elastic waves and $q_{r}$ is the heat flux in the radial direction.

In the non-dimensional variables, the governing equations, equations (13-16) and (22), can be expressed in the following forms (dropping primes for convenience):

$$
\begin{gathered}
\left(1+\frac{c_{3}^{2}}{c_{1}^{2}}+\frac{c_{2}^{2}}{c_{1}^{2}} \frac{\partial}{\partial t}\right) \nabla^{2} e-\left(1+\beta_{1} \frac{\partial}{\partial t}\right) \nabla^{2} \theta \\
-\left(1+\beta_{2} \frac{\partial}{\partial t}\right) \nabla^{2} C=\frac{\partial^{2} e}{\partial t^{2}} \\
\nabla^{2} \theta=\left(\frac{\partial}{\partial t}+t_{0} \frac{\partial^{2}}{\partial t^{2}}\right)\left[\theta+\varepsilon\left(1+\beta_{1} \frac{\partial}{\partial t}\right) e+\varepsilon \eta_{1} C\right] \\
\left(1+\beta_{2} \frac{\partial}{\partial t}\right) \nabla^{2} e+\eta_{1} \nabla^{2} \theta+\eta_{2}\left(\frac{\partial}{\partial t}+t_{1} \frac{\partial^{2}}{\partial t^{2}}\right) C=\eta_{3} \nabla^{2} C \\
\sigma_{r r}=\left(1+\frac{c_{2}^{2}}{c_{1}^{2}} \frac{\partial}{\partial t}\right) e-\frac{4}{\beta^{2}}\left(1+\alpha_{2} \frac{\partial}{\partial t}\right) \frac{u}{r} \\
-\left(1+\beta_{1} \frac{\partial}{\partial t}\right) \theta-\left(1+\beta_{2} \frac{\partial}{\partial t}\right) C \\
\sigma_{\Theta \Theta}=\left(1-\frac{2}{\beta^{2}}\right)\left(1+\alpha_{1} \frac{\partial}{\partial t}\right) e \\
+\frac{2}{\beta^{2}}\left(1+\alpha_{2} \frac{\partial}{\partial t}\right) \frac{u}{r}-\left(1+\beta_{1} \frac{\partial}{\partial t}\right) \theta \\
\quad-\left(1+\beta_{2} \frac{\partial}{\partial t}\right) C \\
P=\eta_{3} C-\left(1+\beta_{2} \frac{\partial}{\partial t}\right) e-\eta_{1} \theta
\end{gathered}
$$

where

$$
\begin{aligned}
\varepsilon & =\frac{\beta_{1 e}^{2} T_{0}}{\rho^{2} c_{E} c_{1}^{2}}, \quad \eta_{1}=\frac{a \rho c_{1}^{2}}{\beta_{1 e} \beta_{2 e}}, \\
\eta_{2} & =\frac{\rho c_{1}^{2}}{\beta_{2 e}^{2} \eta D}, \quad c_{3}=\frac{\mu_{0} H_{0}^{2}}{\rho} \\
\eta_{3} & =\frac{b \rho c_{1}^{2}}{\beta_{2 e}^{2}}, \quad \beta^{2}=\frac{\lambda_{e}+2 \mu_{e}}{\mu_{e}} \\
c_{2}^{2} & =\frac{\lambda_{e} \alpha_{1}+2 \mu_{e} \alpha_{2}}{\rho} .
\end{aligned}
$$

\section{Initial and boundary conditions}

The initial and regularity conditions are given by

$$
\begin{aligned}
& u=0=\frac{\partial u}{\partial t}, \quad \theta=0=\frac{\partial \theta}{\partial t}, \\
& C=0=\frac{\partial C}{\partial t} \quad \text { at } t=0 .
\end{aligned}
$$

The homogeneous initial conditions are supplemented by the following boundary conditions:

(1) The cavity surface is traction free:

$$
\sigma_{r r}+M_{r r}=0 \text { at } r=R .
$$

where $M_{r r}$ is the Maxwell stress in the sphere. 
(2) The cavity surface is subjected to a thermal shock, i.e.,

$$
\theta=\theta_{0} H(t) \text { at } r=R .
$$

(3) The chemical potential is also assumed to be a known function of time at the cavity surface

$$
P=P_{0} H(t) \text { at } r=R \text {. }
$$

where $\theta_{0}$ and $P_{0}$ are constants and $H(t)$ is heaviside unit step function.

\section{Solution of the problem in Laplace transform domain}

Applying Laplace transform defined by

$$
\bar{f}(r, s)=\int_{0}^{\infty} e^{-s t} f(r, t) d t
$$

into equations (24-26) under the initial conditions given in equation (28), one obtains

$$
\begin{gathered}
\left(\nabla^{2}-\omega_{1}\right) \bar{e}=\omega_{2} \nabla^{2} \bar{\theta}+\omega_{3} \nabla^{2} \bar{C}, \\
\left(\nabla^{2}-\omega_{4}\right) \bar{\theta}=\omega_{5} \bar{e}+\omega_{6} \bar{C}, \\
\zeta_{2} \nabla^{2} \bar{e}+\eta_{1} \nabla^{2} \bar{\theta}+\zeta_{5} \bar{C}=\eta_{3} \nabla^{2} \bar{C}, \\
\bar{\sigma}_{r r}=\omega_{0} \bar{e}-2 \zeta_{3} \frac{\bar{u}}{r}-\zeta_{1} \bar{\theta}-\zeta_{2} \bar{C}, \\
\bar{\sigma}_{\Theta \Theta}=\zeta_{4} \bar{e}+\zeta_{3} \frac{\bar{u}}{r}-\zeta_{1} \bar{\theta}-\zeta_{2} \bar{C}, \\
\bar{P}=\eta_{3} \bar{C}-\zeta_{2} \bar{e}-\eta_{1} \bar{\theta},
\end{gathered}
$$

where

$$
\begin{aligned}
& \omega_{1}=\frac{s^{2}}{\omega_{0}}, \quad \omega_{2}=\frac{\zeta_{1}}{\omega_{0}}, \quad \omega_{3}=\frac{\zeta_{2}}{\omega_{0}}, \\
& \omega_{0}=1+\frac{c_{3}^{2}}{c_{1}^{2}}+\frac{c_{2}^{2}}{c_{1}^{2}} s, \quad \zeta_{1}=1+\beta_{1} s, \\
& \zeta_{2}=1+\beta_{2} s, \quad \zeta_{3}=\frac{2\left(1+\alpha_{2} s\right)}{\beta^{2}}, \\
& \zeta_{4}=\frac{1}{\beta^{2}}\left(\beta^{2}-2\right)\left(1+\alpha_{1} s\right), \quad \zeta_{5}=\eta_{2}\left(s+t_{1} s^{2}\right), \\
& \omega_{4}=s+t_{0} s^{2}, \quad \omega_{5}=\omega_{4} \varepsilon\left(1+\beta_{1} s\right), \quad \omega_{6}=\omega_{4} \varepsilon \eta_{1} .
\end{aligned}
$$

Eliminating $\bar{e}, \bar{C}$ between equation (33), one gets six-order partial differential equation satisfied by $\bar{\theta}$ in the form

$$
\left(\nabla^{6}-A_{1} \nabla^{4}+A_{2} \nabla^{2}-A_{3}\right) \bar{\theta}=0,
$$

where

$$
\begin{aligned}
A_{1} & =\frac{y_{2} x_{2}+y_{1} x_{3}-\omega_{1} y_{3}-x_{1} y_{4}}{y_{1} x_{2}-x_{1} y_{3}} \\
A_{2} & =\frac{y_{2} x_{3}-\omega_{1} y_{4}-x_{1} y_{5}}{y_{1} x_{2}-x_{1} y_{3}} \\
A_{3} & =\frac{-\omega_{1} y_{5}}{y_{1} x_{2}-x_{1} y_{3}}
\end{aligned}
$$

in which

$$
\begin{aligned}
& x_{1}=1+\frac{\omega_{3} \omega_{5}}{\omega_{6}}, \quad x_{2}=\frac{\omega_{3}}{\omega_{6}}, \\
& x_{3}=\frac{\omega_{3} \omega_{4}}{\omega_{6}}-\omega_{2}, \quad y_{1}=\zeta_{2}+\frac{\eta_{3} \omega_{5}}{\omega_{6}}, \\
& y_{2}=\frac{\zeta_{5} \omega_{5}}{\omega_{6}}, \quad y_{3}=\frac{\eta_{3}}{\omega_{6}}, \\
& y_{4}=\eta_{1}+\frac{\zeta_{5}}{\omega_{6}}+\frac{\eta_{3} \omega_{4}}{\omega_{6}}, \quad y_{5}=\frac{\zeta_{5} \omega_{5}}{\omega_{6}} .
\end{aligned}
$$

In a similar manner, we can show that $\bar{e}$ and $\bar{C}$ satisfy the equations

$$
\left(\nabla^{6}-A_{1} \nabla^{4}+A_{2} \nabla^{2}-A_{3}\right)\{\bar{e}, \bar{C}\}=0 .
$$

Introducing $m_{i}(i=1,2)$ into equation (37), one gets

$$
\left(\nabla^{2}-m_{1}^{2}\right)\left(\nabla^{2}-m_{2}^{2}\right)\left(\nabla^{2}-m_{3}^{2}\right) \bar{\theta}=0,
$$

where $m_{1}^{2}, m_{2}^{2}$ and $m_{3}^{2}$ are the positive solutions for the characteristic equation

$$
m^{6}-A_{1} m^{4}+A_{2} m^{2}+A_{3}=0 .
$$

The roots $m_{1}, m_{2}$ and $m_{3}$ are given by

$$
\begin{aligned}
m_{1} & =\sqrt{\frac{1}{3}\left[2 p \sin (q)+A_{1}\right]}, \\
m_{2} & =\sqrt{-\frac{p}{3}[\sqrt{3} \cos (q)+\sin (q)]+\frac{A_{1}}{3}}, \\
m_{3} & =\sqrt{\frac{p}{3}[\sqrt{3} \cos (q)-\sin (q)]+\frac{A_{1}}{3}},
\end{aligned}
$$

where

$$
\begin{aligned}
& p=\sqrt{A_{1}^{2}-3 A_{2}}, \quad q=\frac{1}{3} \sin ^{-1}(\chi), \\
& \chi=-\frac{2 A_{1}^{3}-9 A_{1} A_{2}+27 A_{3}}{2 p^{3}} .
\end{aligned}
$$

The solution of equation (41), which is bounded at infinity, is given by

$$
\bar{\theta}(r, s)=\frac{1}{\sqrt{r}} \sum_{i=1}^{3} B_{i}(s) K_{1 / 2}\left(m_{i} r\right),
$$

where $B_{i}$ are parameters depending on $s$ and $K_{1 / 2}(\cdot)$ are the modified Bessel function of the second kind of order $1 / 2$. 
In a similar manner, one can write

$\{\bar{e}(r, s), \bar{C}(r, s)\}=\frac{1}{\sqrt{r}} \sum_{i=1}^{3}\left\{B_{i}^{\prime}(s), B_{i}^{\prime \prime}(s)\right\} K_{1 / 2}\left(m_{i} r\right)$.

Substituting equation (46) into equations (33) and (34), one gets the following relations:

$$
\begin{aligned}
B_{i}^{\prime} & =\frac{y_{3} m_{i}^{4}-y_{4} m_{i}^{2}+y_{5}}{y_{1} m_{i}^{2}-y_{2}} B_{i}=\Gamma_{i} B_{i}, \\
B_{i}^{\prime \prime} & =\frac{\left(y_{1}-\omega_{5} y_{3}\right) m_{i}^{4}-\left(y_{1} \omega_{4}+y_{2}-y_{4} \omega_{5}\right) m_{i}^{2}}{+\omega_{4} y_{2}-\omega_{5} y_{5}} \\
& =\Omega_{6} B_{i} .
\end{aligned}
$$

Thus, one gets

$$
\{\bar{e}(r, s), \bar{C}(r, s)\}=\frac{1}{\sqrt{r}} \sum_{i=1}^{3}\left\{\Gamma_{i}, \Omega_{i}\right\} B_{i} K_{1 / 2}\left(m_{i} r\right) .
$$

Using the relation between $\bar{u}$ and $\bar{e}$, one gets the solution for the dimensionless form of displacement assuming that $\bar{u}$ vanishes at infinity as:

$$
\bar{u}=-\frac{1}{\sqrt{r}} \sum_{i=1}^{3} \frac{\Gamma_{i}}{m_{i}} B_{i}(s) K_{3 / 2}\left(m_{i} r\right) .
$$

Thus, from equations (48) and (49), one gets

$$
\begin{aligned}
& \left\{\begin{array}{c}
\bar{\sigma}_{r r}(r, s) \\
\bar{\sigma}_{\Theta \Theta}(r, s) \\
\bar{P}(r, s)
\end{array}\right\}=\frac{1}{\sqrt{r}} \sum_{i=1}^{3} B_{i}(s) \\
& \quad \times\left[\begin{array}{cc}
\omega_{0} \Gamma_{i}-\zeta_{1}-\zeta_{2} \Omega_{i} & \frac{2 \zeta_{3} \Gamma_{i}}{m_{i} r} \\
\zeta_{4} \Gamma_{i}-\zeta_{1}-\zeta_{2} \Omega_{i} & -\frac{\zeta_{3} \Gamma_{i}}{m_{i} r} \\
\eta_{3} \Omega_{i}-\eta_{1}-\zeta_{2} \Gamma_{i} & 0
\end{array}\right]\left\{\begin{array}{l}
\left.K_{1 / 2}\left(m_{i} r\right)\right\} \\
\left.K_{3 / 2}\left(m_{i} r\right)\right\}
\end{array}\right] .
\end{aligned}
$$

The transformed boundary conditions become

$$
\begin{aligned}
& \bar{\sigma}_{r r}=0, \quad \bar{\theta}=\theta_{0} / s, \\
& \bar{P}=P_{0} / s, \quad \text { at } r=R .
\end{aligned}
$$

In order to evaluate the unknown parameters $B_{i}$, Laplace transform of the boundary conditions given in equation (51) together with equations (46) and (50) is used. Thus, one arrives to the following set of linear equations:

$$
\begin{aligned}
& \sum_{i=1}^{3} B_{i}(s) K_{1 / 2}\left(m_{i} R\right)=\frac{\theta_{0} \sqrt{R}}{s}, \\
& \sum_{i=1}^{3}\left(\left(\omega_{0} \Gamma_{i}-\zeta_{1}-\zeta_{2} \Omega_{i}\right) K_{1 / 2}\left(m_{i} R\right)\right. \\
& \left.\quad+\frac{2 \zeta_{3} \Gamma_{i}}{m_{i} R} K_{3 / 2}\left(m_{i} R\right)\right) B_{i}(s)=0,
\end{aligned}
$$

$$
\begin{aligned}
\sum_{i=1}^{3}( & \left.\eta_{3} \Omega_{i}-\eta_{1}-\zeta_{2} \Gamma_{i}\right) K_{1 / 2}\left(m_{i} R\right) B_{i}(s) \\
= & \frac{P_{0} \sqrt{R}}{s} .
\end{aligned}
$$

The above equations can be written in the matrix form $\mathbf{A X}=\mathbf{Z}$, where $\mathbf{A}=\left(a_{i j}\right)$ is the $3 \times 3$ matrix coefficients of the system, $\mathbf{X}=\left\{B_{1}, B_{2}, B_{3}\right\}^{T}$ and $\mathbf{Z}=\left\{\frac{\theta_{0} \sqrt{R}}{s}, 0, \frac{P_{0} \sqrt{R}}{s}\right\}^{T}$. The solution of this system gives the parameters $B_{1}, B_{2}$ and $B_{3}$. This completes the solution of the problem in Laplace transform domain.

\section{Special cases}

\subsection{Generalized magneto-thermoelastic theory with diffusion}

Neglecting the viscous effect by taking $\alpha_{1}=\alpha_{2}=0$. So, the expressions for the displacement components, stresses and temperature field can be obtained in the generalized magneto-thermoelastic with diffusion heat conduction equation.

\subsection{Generalized thermoelastic theory with diffusion}

Neglecting the viscous effect by taking $\alpha_{1}=\alpha_{2}=0$ and neglecting the magnetic field by setting $c_{3}=0$. The expressions for the displacement components, stresses and temperature field can be obtained in the generalized thermoelastic with diffusion heat conduction equation.

\subsection{Generalized magneto-thermoviscoelastic theory with one relaxation time}

The generalized magneto-thermoviscoelastic theory with one relaxation time can be deduced by putting the concentration of the diffusive material $C=0$ and the diffusion coefficient $D=0$.

\subsection{Generalized thermoviscoelastic theory with one relaxation time}

The generalized thermoviscoelastic theory with one relaxation time can be deduced by putting the concentration of the diffusive material $C=0$, the diffusion coefficient $D=0$ and magnetic field $c_{3}=0$.

\subsection{Generalized magneto-thermoelastic theory with one relaxation time}

Neglecting the viscous effect and the effect of the diffusion by taking $\alpha_{1}=\alpha_{2}=0$ and $C=D=0$ 
to obtain the expressions for the displacement components, stresses and temperature field in the generalized magneto-thermoelastic theory.

\subsection{Generalized thermoelastic theory with one relaxation time}

Neglecting the viscous effect, the effect of the diffusion and the magnetic field by taking $\alpha_{1}=\alpha_{2}=0$, $C=D=0$ and $c_{3}=0$, respectively. One obtains the expressions for the displacement components, stresses and temperature field in the generalized thermoelastic theory.

\section{Inversion of Laplace transforms}

To obtain a solution of the problem in the physical domain, the transforms in equations (41), (45), (46) and (48-50) are inverted. In order to invert Laplace transform in the above equations, we adopt a numerical inversion method based on a Fourier series expansion (Honig and Hirdes 1984). In this method, the inverse $g(t)$ of Laplace transform $\bar{g}(s)$ is approximated by the relation

$$
\begin{aligned}
& g(t)=\frac{\mathrm{e}^{c t}}{t_{1}}\left(\frac{g(c)}{2}+\operatorname{Re} \sum_{k=1}^{N} \mathrm{e}^{i k \pi t / t_{1}} g\left(c+i k \pi / t_{1}\right)\right), \\
& 0 \leq t \leq t_{1},
\end{aligned}
$$

where $N$ is a sufficiently large integer representing the number of terms in the truncated infinite Fourier series. It must be chosen such that

$$
\mathrm{e}^{c t} \operatorname{Re}\left\{\mathrm{e}^{i N \pi t / t_{1}} g\left(c+i N \pi / t_{1}\right)\right\} \leq \varepsilon_{1},
$$

where $\varepsilon_{1}$ is a persecuted small positive number that corresponds to the degree of accuracy to be achieved. $c$ is a positive free parameter that must be greater than the real parts of all singularities of $\bar{g}(s)$. The optimal choice of $c$ was obtained according to the criteria described in Honig and Hirdes (1984).

Two methods are used to reduce the total error. First, Korrecktur method is used to reduce the discretization error. Next, the e-algorithm is used to reduce the truncation error and hence to accelerate convergence. It should be noted that a good choice of the free parameters $N$ and $c t$ is not only important for the accuracy of the results but also for the application of Korrecktur method and the methods for the acceleration of convergence.

\section{Numerical example and discussion}

In order to discuss the problem in great detail and to find out the nature of dependence of physical variables on diffusion and viscosity, we have computed them numerically for a particular model. Copper material was chosen for the purpose of numerical evaluation. The material properties of the problem are thus given in SI units (Kanoria and Mallik 2010) as:

$$
\begin{aligned}
& T_{0}=293 \mathrm{~K}, \quad \rho=8954 \mathrm{~kg} \mathrm{~m}^{-3}, \quad \tau_{0}=0.02 \mathrm{~s} \\
& \tau=0.2 \mathrm{~s}, \quad \alpha_{1}=0.06 \mathrm{~s}, \quad \alpha_{2}=0.09 \mathrm{~s} \\
& \alpha_{t}=1.78 \times 10^{-5} \mathrm{~K}^{-1}, \\
& \alpha_{c}=1.98 \times 10^{-4} \mathrm{~m}^{3} \mathrm{~kg}^{-1} \\
& \mu=3.86 \times 10^{10} \mathrm{~kg} \mathrm{~m}^{-1} \mathrm{~s}^{-2}, \lambda=7.76 \times 10^{10} \mathrm{~kg} \mathrm{~m}^{-1} \mathrm{~s}^{-2} \\
& C_{E}=383.1 \mathrm{~J} \mathrm{~kg}^{-1} \mathrm{~K}^{-1}, K=386 \mathrm{~W} \mathrm{~m}^{-1} \mathrm{~K}^{-1} \\
& D=0.85 \times 10^{-8} \mathrm{~kg} \mathrm{~s}^{-3}, \\
& a=1.2 \times 10^{4} \mathrm{~m}^{2} \mathrm{~s}^{-2} \mathrm{~K}^{-1}, \\
& b=0.9 \times 10^{6} \mathrm{~m}^{5} \mathrm{~kg}^{-1} \mathrm{~s}^{-2}, \quad \mu_{0}=4 \pi \times 10^{-7} \mathrm{H} \mathrm{m}^{-1} \\
& H_{0}=10^{7} / 4 \pi \mathrm{A} \mathrm{m}^{-1} .
\end{aligned}
$$

The numerical technique outlined above is used to obtain the temperature, radial displacement, radial stress and concentration as well as the chemical potential distributions inside the sphere. The spherical cavity is considered with radius $r=1$ and its center at the origin.

Comparison of the dimensionless physical quantities are made for three different cases: (i) magnetothermoviscoelastic solid with diffusion MTVED, (ii) magneto-thermoelastic solid with diffusion MTED, and (iii) magneto-thermoelastic solid MTE and are shown in figures $1-5$ for $t=0.15$. It can be seen from figure 1 that diffusion acts to decrease the magnitude of the temperature distribution.

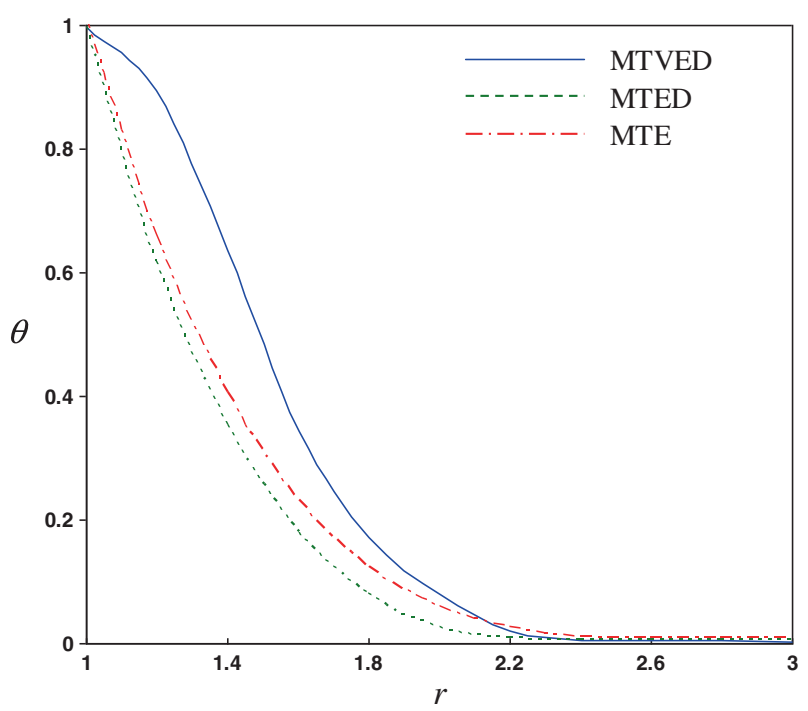

Figure 1. Variation of temperature distribution with distance using different theories. 
The temperature distribution $\theta(r, t)$ in MTVED theory is large compared to MTE and MTED theories. According to all theories, the values of temperature are same on the boundary. It can be noticed that the temperature function approaches zero in the presence of a diffusion effect more rapidly than in the absence of a diffusion effect as the distance $r$ increases.

It is also observed from figure 2 that due to the presence of viscosity term, the displacement $u(r, t)$ has appreciably decreased for viscous case in comparison with non-viscous cases for most values of $r$. The thermoviscoelastic relaxation times $\left(\alpha_{1}\right.$ and $\alpha_{2}$ ) have a small effect on the values of displacement; actually, the displacement increases in the case of MTE theory but decreases in the case of

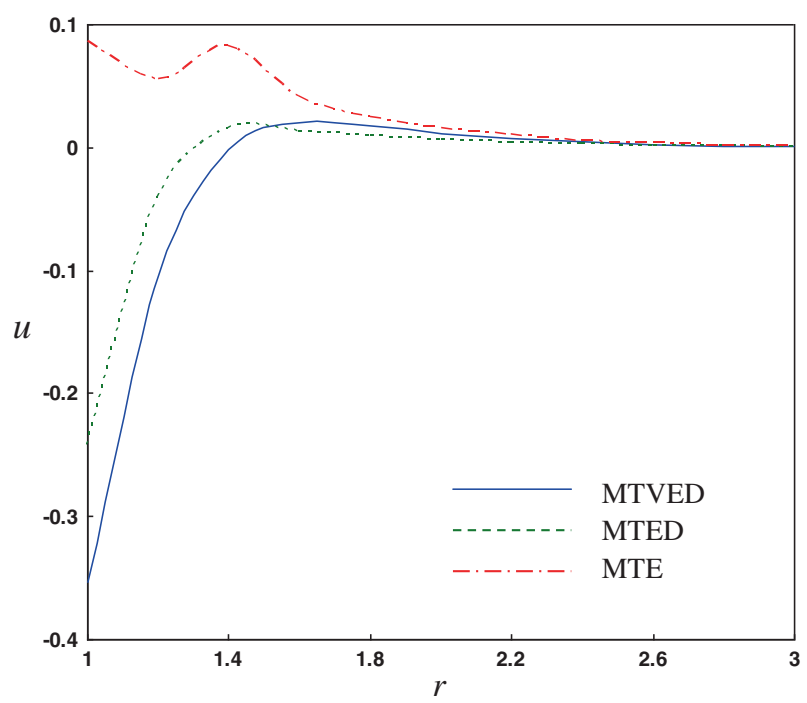

Figure 2. Variation of displacement distribution with distance using different theories.

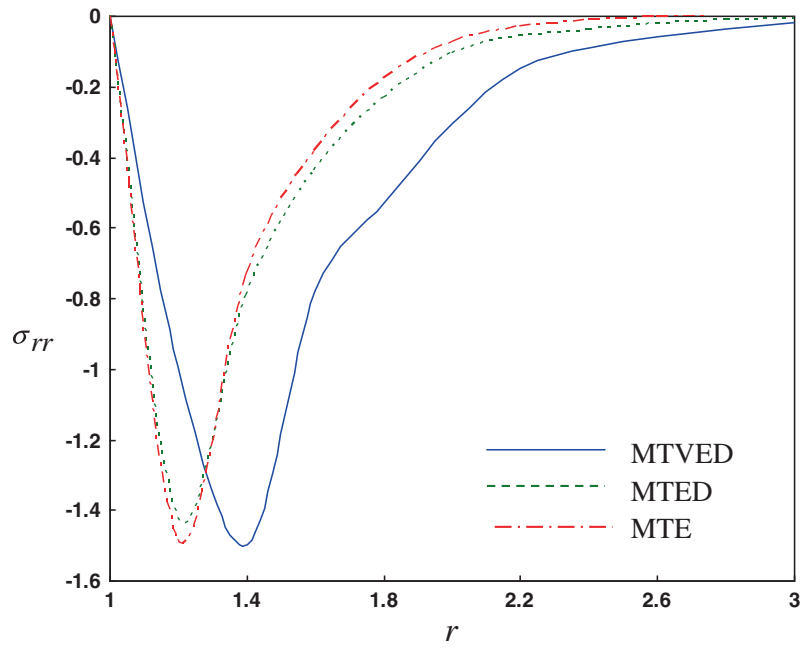

Figure 3. Variation of radial thermal stress distribution with distance using different theories.
MTVED theory. It is to be noted that the absence of viscosity term is responsible for lowering down the values of displacement component and this coincided with the results reported in Deswal and Kalkal (2011). The displacement starts with negative values in case of MTVED and MTED theories while it starts with positive value in case of MTE theory, i.e., the behaviour of displacement in MTE theory is opposite in nature to that of MTVED and MTED theories owing to the absence of viscosity term.

Figure 3 shows that the numerical values of radial stress $\sigma_{r r}(r, t)$ in MTE theory are larger than those in MTVED and MTED theories, especially for $r \geq 1.3$. Figures 4 and 5 represent the change in the concentration $C(r, t)$ about the initial

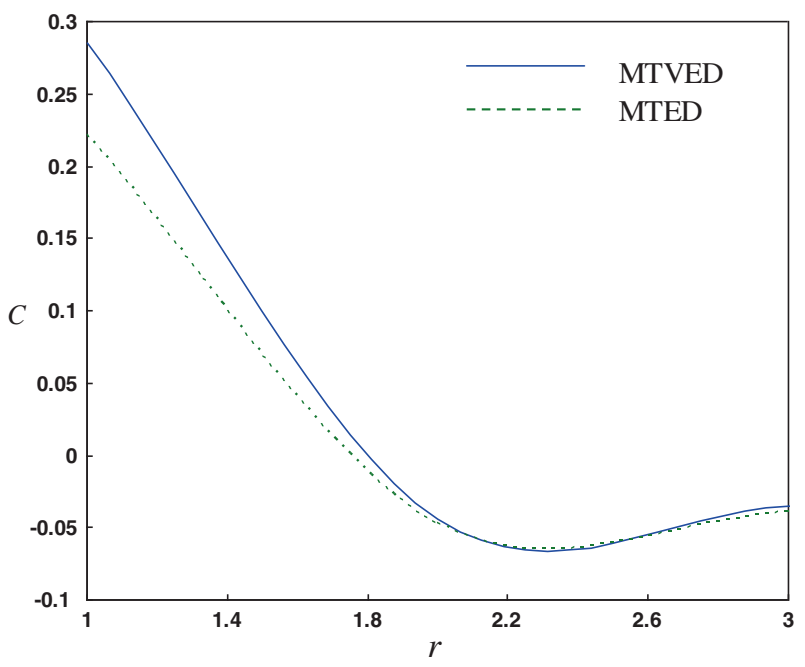

Figure 4. Variation of concentration of the diffusive material distribution with distance using different theories.

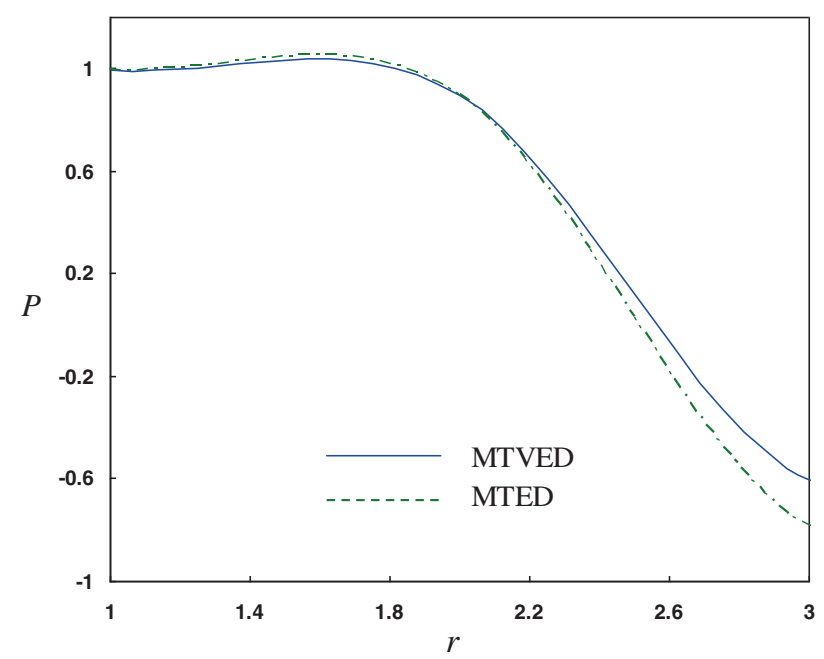

Figure 5. Variation of chemical potential distribution with distance using different theories. 
concentration and the chemical potential $P(r, t)$ along the radial direction. The behaviour of the concentration and the chemical potential for both theories MTVED and MTED are alike. The influence of diffusion factor on various functions is noticed to be quite significant and the functions follow similar trend and behaviour in the presence and absence of diffusion. The maximum impact zone of this factor is near the boundary of medium and their effects lessen as $r$ diverges from the point of source application (see Kalkal and Deswal 2014).

The temperature $\theta(r, t)$, radial displacement $u(r, t)$, radial stress $\sigma_{r r}(r, t)$ and concentration $C(r, t)$ as well as the chemical potential $P(r, t)$ distributions inside the sphere are obtained according to the general case. These distributions are shown in figures 6-10 respectively. The computations were

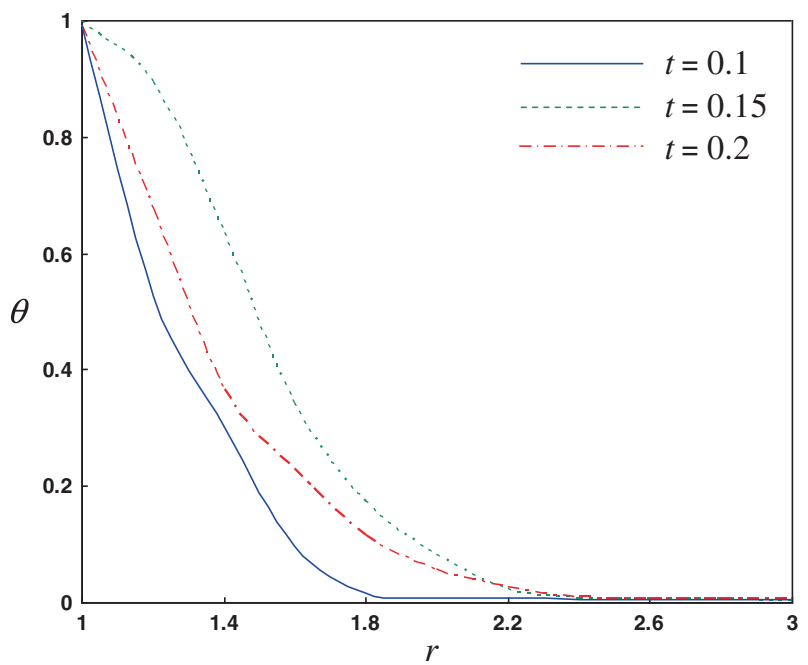

Figure 6. Variation of temperature distribution with distance for three values of time.

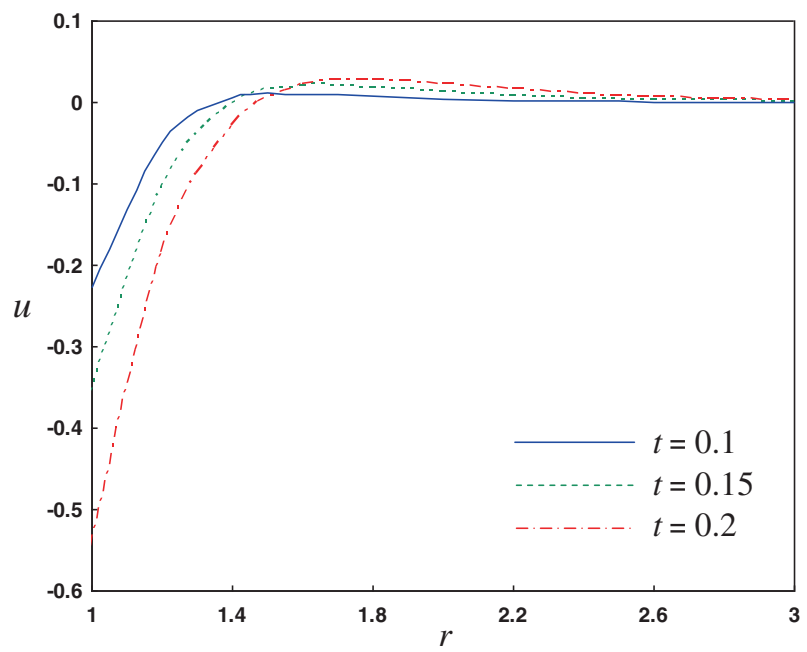

Figure 7. Variation of displacement distribution with distance for three values of time. carried out for three values of time, namely $t=0.1$, $t=0.15$ and $t=0.2$. Figure 6 shows that the value of $\theta$ does not necessarily increase with the increase of time $t$. Figure 7 shows that $u$ decreases as $t$ increases for $r \leq 1.5$ and vice versa for $r \geq 1.5$. Figure 8 shows that the values of $\sigma_{r r}$ decrease with an increase of time along the radial direction. Figure 9 shows that the concentration distribution $C(r, t)$ does not necessarily decrease with the increase of time $t$. The behaviours of $C$ are similar for different values of $t$. Figure 10 shows that the chemical potential $P(r, t)$ decreases with the increase of time $t$. The behaviour of $P$ for $t=0.1$ may differ from that of $t=0.15$ and $t=0.2$.

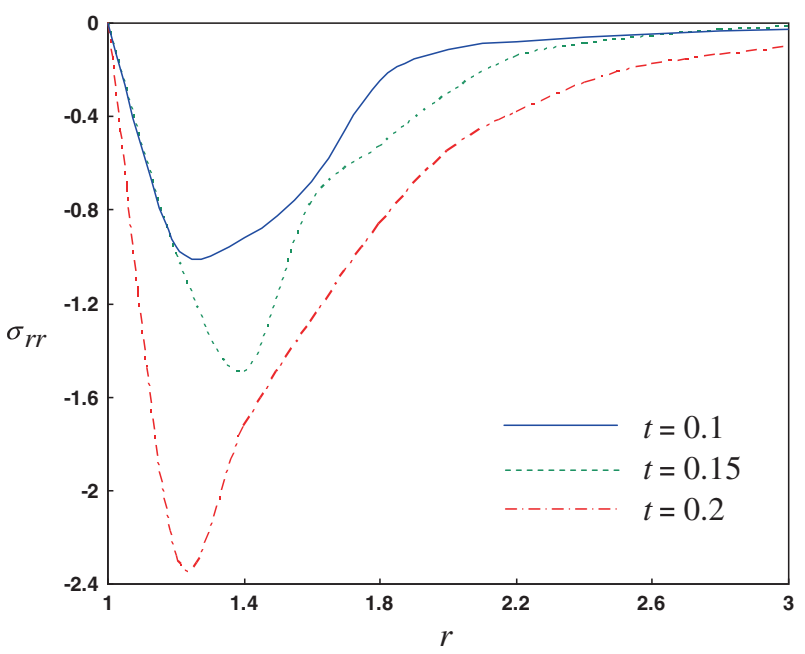

Figure 8. Variation of radial thermal stress distribution with distance for three values of time.

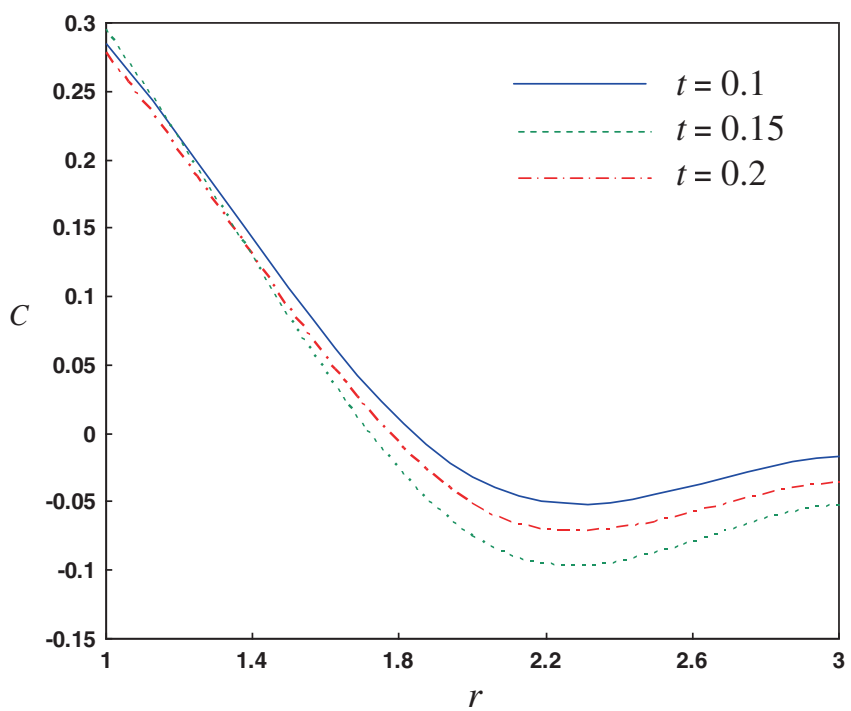

Figure 9. Variation of concentration of the diffusive material distribution with distance for three values of time. 


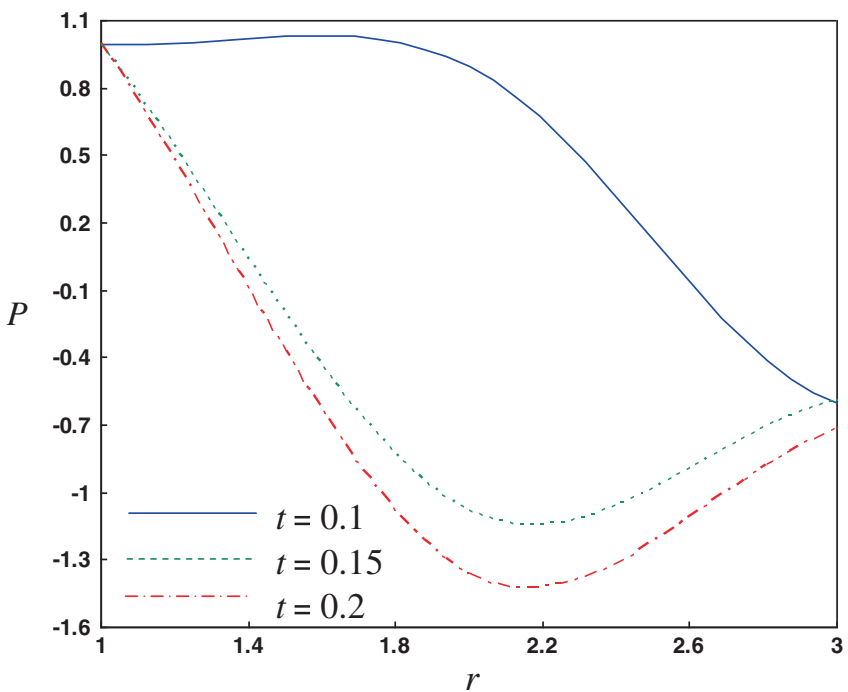

Figure 10. Variation of chemical potential distribution with distance for three values of time.

\section{Conclusions}

The phenomenon of a finite speed of propagation is manifested in all figures. This is different from the cases in both the uncoupled and coupled theories of thermoelasticity where an infinite speed of propagation is inherent, and hence all the considered functions have non-zero values for any point in the medium.

Due to the complicated nature of the governing equations for the generalized thermoviscoelastic diffusion theory, the work done in this field is very limited. The method used in this study provides a quite successful approach in dealing with such problems (Honig and Hirdes 1984). This approach gives exact solutions in the Laplace domain without any assumed restrictions on the actual physical quantities that appear in the governing equations of the problem considered.

It can be observed that the viscous effect plays an important role. It is more pronounced in the fractional order thermoviscoelasticity. Some difference in the value of distributions is noticed for the three theories, i.e., $\alpha_{1}, \alpha_{2}$ and $\gamma_{0}$ have a clear effect. It can also be observed that the diffusion effect plays an important role.

From the figures, it is clear that diffusion strongly affects the physical quantities. It acts to decrease the amplitudes of displacement and stress distributions while it acts to increase the amplitude of the temperature distribution. This is due to the influences of cross effects arising from the coupling of the fields of temperature, mass diffusion and strain. These results are compared well with those of Deswal and Kalkal (2011). The method used in the present study is applicable to a wide range of problems in thermodynamics (Sherief and Saleh 2005; Xia et al. 2009; Zenkour et al. 2012).

Finally, the results obtained in this study should prove useful for researchers in material science, designers of new materials, low-temperature physicists, as well as for those working on the development of a theory of hyperbolic magnetothermo-diffusion. Study of the phenomenon of diffusion is also used to improve the conditions of oil extraction (seeking ways of more efficiently recovering deposits) (Singh 2005). Cross-effects of heat and mass diffusion exchange with the environment arising from and inside nuclear reactors influence their design and operations (Nowinski 1978). The problem of generalized thermoelasticity has been reduced as a special case of our problem.

\section{Acknowledgements}

This work was funded by the Deanship of Scientific Research (DSR), King Abdulaziz University, Jeddah, under grant no. 142/130/1433. The authors acknowledge with thanks DSR technical and financial support.

\section{References}

Banerjee S and Roychoudhuri S K 1995 Spherically symmetric thermo-viscoelastic waves in a visco-elastic medium with a spherical cavity; Comput. Math. Appl. 30 91-98.

Deswal S and Kalkal K 2011 A two-dimensional generalized electro-magneto-thermoviscoelastic problem for a half-space with diffusion; Int. J. Therm. Sci. 50 749-759.

Ezzat M A 1997 Generation of generalized magnetothermoelastic waves by thermal shock in a perfectly conducting half-space; J. Therm. Stress. 20 617-633.

Ezzat M A and El-Karamany A S 2002 The uniqueness and reciprocity theorems for generalized thermoviscoelasticity with two relaxation times; Int. J. Eng. Sci. $401275-1284$.

Green A E and Lindsay A 1972 Thermoelasticity; J. Elasticity 2 1-7.

Honig G and Hirdes U 1984 A method for the numerical inversion of the Laplace transform; J. Comput. Appl. Math. 10 113-132.

Huilgol R and Phan-Thien N 1997 Fluid Mechanics of Viscoelasticity; Elsevier, Amsterdam.

Ilioushin A A and Pobedria B E 1970 Fundamentals of the Mathematical Theory of Thermal Viscoelasticity; Nauka, Moscow.

Kalkal K K and Deswal S 2014 Analysis of vibrations in fractional order magneto-thermo-viscoelasticity with diffusion; J. Mech. 30 383-394.

Kanoria M and Mallik S H 2010 Generalized thermoviscoelastic interaction due to periodically varying heat source with three-phase-lag effect; Eur. J. Mech. A/Solid. 29 695-703.

Lord H and Shulman Y 1967 A generalized dynamical theory of thermoelasticity; J. Mech. Phys. Solid. 15 299-309.

Nowacki W 1974a Dynamical problems of thermodiffusion in solids I; Bull. Acad. Pol. Sci. Ser. Sci. Tech. 22 55-64.

Nowacki W 1974b Dynamical problems of thermodiffusion in solids II; Bull. Acad. Pol. Sci. Ser. Sci. Tech. 22 129-135. 
Nowacki W 1974c Dynamical problems of thermodiffusion in solids III; Bull. Acad. Pol. Sci. Ser. Sci. Tech. 22 266275 .

Nowinski J L 1978 Theory of Thermoelasticity with Applications; Sijthoff \& Noordhoff International Alphen Aan Den Rijn.

Roychoudhuri S K and Banerjee S 1998 Magnetothermoelastic interactions in an infinite viscoelastic cylinder of temperature rate dependent material subjected to a periodic loading; Int. J. Eng. Sci. 36 635-643.

Roychoudhuri S K and Mukhopadhyay S 2000 Effect of rotation and relaxation times on plane waves in generalized thermo-viscoelasticity; Int. J. Math. Math. Sci. 23 497-505.

Sherief H H and Saleh H 2005 A half-space problem in the theory of generalized thermoelastic diffusion; Int. J. Solids. Struct. 42 4484-4494.
Sherief H H, Hamza F and Saleh H 2004 The theory of generalized thermoelastic diffusion; Int. J. Eng. Sci. 42 591-608.

Singh B 2005 Reflection of P and SV waves from free surface of an elastic solid with generalized thermodiffusion; J. Earth Syst. Sci. 114 159-168.

Tanner R I 1988 Engineering rheology; Oxford University Press, Oxford.

Xia R, Tian X and Shen Y 2009 The influence of diffusion on generalized thermoelastic problems of infinite body with a cylindrical cavity; Int. J. Eng. Sci. 47 669-679.

Zenkour A M, Mashat D S and Abouelregal A E 2012 Generalized thermodiffusion for an unbounded body with a spherical cavity subjected to periodic loading; J. Mech. Sci. Tech. 26 749-757. 